\title{
Bulk metals with helical surface states
}

\author{
Doron L. Bergman and Gil Refael \\ Physics Department, California Institute of Technology, MC 114-36, 1200 E. California Boulevard, Pasadena, California 91125, USA
}

(Received 21 May 2010; revised manuscript received 13 October 2010; published 9 November 2010)

\begin{abstract}
In the flurry of experiments looking for topological insulator materials, it has been recently discovered that some bulk metals very close to topological insulator electronic states support the same topological surface states that are the defining characteristic of the topological insulator. First observed in spin-polarized angle resolved photoemission spectroscopy (ARPES) in Sb [D. Hsieh et al., Science 323, 919 (2009)], the helical surface states in the metallic systems appear to be robust to at least mild disorder. We present here a theoretical investigation of the nature of these "helical metals"-bulk metals with helical surface states. We explore how the surface and bulk states can mix, in both clean and disordered systems. Using the Fano model, we discover that in a clean system, the helical surface states are not simply absorbed by hybridization with a nontopological parasitic metallic band. Instead, they are pushed away from overlapping in momentum and energy with the bulk states, leaving behind a finite-lifetime surface resonance in the bulk energy band. Furthermore, the hybridization may lead in some cases to multiplied surface-state bands, in all cases retaining the helical characteristic. Weak disorder leads to very similar effects—surface states are pushed away from the energy bandwidth of the bulk, leaving behind a finite-lifetime surface resonance in place of the original surface states.
\end{abstract}

DOI: 10.1103/PhysRevB.82.195417

PACS number(s): 73.20.At

\section{INTRODUCTION}

The prediction and subsequent discovery of bulk topological insulators (TIs) has galvanized the condensed-matter community, not in the least because of the unique physics of the protected edge states on their surface. ${ }^{1-27}$ Many materials adjacent to TIs, however, are bulk metals with the same helical surface states, e.g., $\quad \mathrm{Sb}_{,}{ }^{28,29} \quad \mathrm{Bi}_{0.91} \mathrm{Sb}_{0.09},{ }^{30,31}$ $\mathrm{Bi}_{2-x} \mathrm{Mn}_{x} \mathrm{Te}_{3},{ }^{32}$ and even undoped $\mathrm{Bi}_{2} \mathrm{Se}_{3},{ }^{33,34}$ which initially was thought to be a wide band-gap TI but now seems to have a small but measurable bulk Fermi surface. In such materials one would naively expect that a bulk Fermi surface would simply swallow the surface states. Nevertheless, the same helical surface states of the TI phase, continue to appear in them, now coexisting with a bulk Fermi surface. We suspect that more examples will emerge, especially since the helical surface states are less sensitive to disorder than ordinary (nonhelical) surface states (of the likes of those found on the $\langle 111\rangle$ surfaces of $\mathrm{Cu}$ ), as has been already suggested by surface-doping $\mathrm{Sb}$ with potassium. ${ }^{28}$

The prevalence of metallic systems with surviving helical surface states led us to ask: what exactly happens when a surface state, which is the result of a TI bulk, is allowed to hybridize with a "parasitic" nontopological metallic band. As we shall see below, not only do the helical edge states survive but they can actually multiply. When mixed, the surface states are simply pushed away from overlapping in energy and momentum with the bulk states. If the surface and bulk do not overlap to begin with, the mixing is not effective, and the surface states are only slightly modified. In those areas of energy and momentum overlap between the bulk and surface states, new "exiled" surface states appear above and below the confines of the metallic band, and in place of the original surface state a "ghost" surface resonance remains, with a finite (and often very short) lifetime. Thus in one momentum value, we may see ARPES signatures of two surface states at energies above, and below, the metallic energy range (see
Fig. 1), in addition to a surface resonance within the bulk metallic band. The exiled states, as well as the remaining finite-lifetime ghost resonances will retain the odd-number distinction of surface bands, characteristic of helical surface states, comprising an odd number of Kramer's pairs of bands in two dimensions (2D) and an odd number of Dirac cones in three dimensions (3D).

In this paper, we explore the metal vs edge-state struggle by first constructing 2D examples where a metallic band appears at the same energy as an edge state, and numerically investigating its emerging spectral structure. Next, we approach the problem analytically by constructing a generic model for a helical surface state interacting with a bulk metal, based on the Fano model. ${ }^{35}$ From both approaches, the

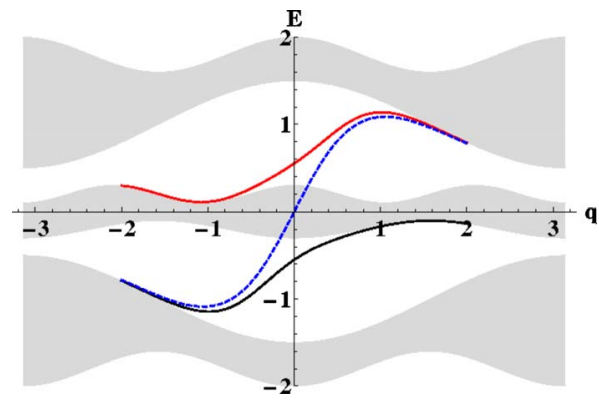

FIG. 1. (Color online) An energy-momentum sketch of the fate of surface states when they overlap with a parasitic metallic bulk band. The solid (light green) regions mark the bulk density of states, the top and bottom bands being bulk states of a topological insulator, and a parasitic metallic band in the middle. The original surface-state branch (dashed line) overlaps with the middle (metallic) band. The surface-state branch hybridizes with the bulk, leaving a diffuse resonance in the bulk band energy range, as well as two sharp edge-state branches appearing above and below the parasitic metal energies. This demonstrates that the topological surface states, rather than being obliterated, are simply pushed away from overlapping with the bulk. 
generic picture of a helical metal arises, as is summarized in Fig. 1.

\section{CONSTRUCTION OF 2D HELICAL METALS}

We begin our study by attempting to construct disorder free-theoretical models in $2 \mathrm{D}$ where helical edge states coexist with a bulk metal in both momentum and energy. Such helical metals can be achieved in at least two different ways. One possible construction is to take a model of a TI, add a new (initially decoupled) partially filled band, and then mix it with the TI bands. A second possible construction is to add a momentum-dependent chemical potential without adding additional degrees of freedom, such that the gap closes somewhere in the Brillouin zone (BZ). This can always be achieved in a tight-binding model by adding appropriate hopping terms that produce purely diagonal terms in the multiband hopping model. We will consider an explicit example of the former, demonstrating the construction in 2D. An explicit example of the latter construction is relegated to Appendix A, as the main physical features of it are no different than in the one example we show here. Our 2D examples can easily be generalized for models of topological insulators in any dimension.

Our starting point is the first 2D model of a topological insulator, the Kane-Mele model, ${ }^{1,3}$ defined on a honeycomb lattice. The first construction proposed above can be realized by considering this model coupled to a very simple metallic band: we add lattice sites at the centers of the honeycomb plaquettes, as shown in Fig. 2, so that the new sites are now those of a triangular lattice. To form a metallic band we allow hopping between the nearest-neighboring new (triangular) sites. With a Fermi energy crossing anywhere in this band, we have a single Fermi surface, centered about $\mathbf{q}=0$. We then couple between the two parts of our model, by allowing hopping between the honeycomb lattice sites and the new triangular lattice sites (see Fig. 2). The Hamiltonian is

$$
\begin{aligned}
\mathcal{H}= & -t_{1} \sum_{\langle i j\rangle \alpha}\left[a_{i \alpha}^{\dagger} b_{j \alpha}+\text { H.c. }\right]-t_{2} \sum_{\langle i j\rangle \alpha}\left[c_{i \alpha}^{\dagger} c_{j \alpha}+\text { H.c. }\right] \\
& +i \lambda \sum_{\langle\langle i j\rangle\rangle \alpha \beta}\left[a_{i \alpha}^{\dagger} a_{j \beta} s_{\alpha \beta}^{z} \nu_{i j}+\text { H.c. }+(a \rightarrow b)\right] \\
& -t_{3} \sum_{\langle i j\rangle \alpha}\left[c_{i \alpha}^{\dagger} a_{j \alpha}+c_{i \alpha}^{\dagger} b_{j \alpha}+\text { H.c. }\right]+h \sum_{j} c_{j}^{\dagger} c_{j},
\end{aligned}
$$

where $i, j$ denote all the combined lattice sites, $a, b, c$ denote the fermion operators on the three sublattices $(a, b$ for the honeycomb, and $c$ for the triangular metal, see Fig. 2). In addition, spin indices are denoted by $\alpha \beta$, and $\nu_{i j}$ is as defined in Refs. 1 and 3 (and shown in Fig. 2). For convenience we have included an independent chemical potential $h$ for the triangular lattice.

Following closely the procedure in Refs. 1 and 3, we calculate the spectrum of Eq. (1) using exact diagonalization of strips that are terminated either at a zigzag or an armchair edge, as these are representative edge cuts of generic boundaries of the honeycomb lattice. We calculate for one spin polarization in order to avoid clutter, as $s^{z}$ is a good quantum

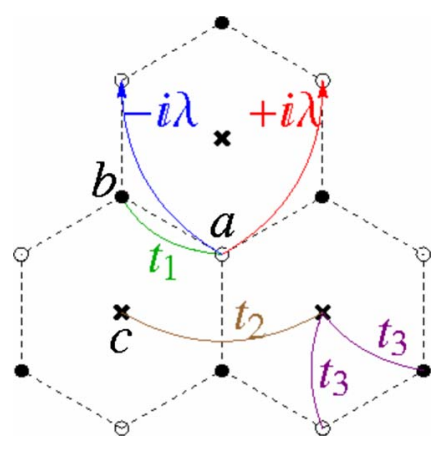

FIG. 2. (Color online) A two-dimensional toy model for the investigation of a helical metal. The model is based on the KaneMele model (Refs. 1 and 3) on the honeycomb lattice, which is denoted by dashed lines. The Kane-Mele model consists of nearestneighbor hopping $t_{1}$ (green) between the two $(a, b)$ sublattices of the honeycomb lattice (denoted by empty and filled circles, respectively), and a complex second-neighbor hopping (a spin-orbit coupling term) with opposite sign when clockwise $(-i \lambda$, blue) and counterclockwise $(+i \lambda$, red), as indicated by the curved arrows in the figure. In addition to sites of the honeycomb lattice, we include a new set of sites $(c)$ at the centers of the hexagonal plaquettes of the honeycomb lattice, denoted by cross marks. The $c$ sites form a triangular sublattice, and nearest-neighbor hopping between them $t_{2}$ (brown) forms a metallic band. To explore the interplay between the topological insulators helical surface states and the bulk metallic band we mix the two systems by allowing hopping between the $c$ sites and the honeycomb lattice sites, $t_{3}$ (purple).

number in our model, and deviations from this are immaterial to the physics we explore here, and identify explicitly the eigenstates localized at each edge of the finite strip. We present the spectrum of the model in Fig. 3, when the honeycomb-triangular hybridization parameter is zero, weak, and strong $\left(t_{3}=0,0.03\right.$, and 0.3 , respectively) while keeping the other model parameters fixed at $t_{1}=1, \lambda=0.5, t_{2}=0.2$, and $h=0.4$. We choose the Bravais lattice vectors to be $\mathbf{a}_{1}=a \hat{x}$ and $\mathbf{a}_{2}=\frac{a}{2}(-\hat{x}+\sqrt{3} \hat{y})$. The strip widths we use are 30 unit cells and are terminated in a symmetric fashion. For the zigzag geometry, states identified as edge states on one side of the system have at least 0.85 of their total weight within a distance of $y=30 \frac{a}{4}$ from the lower edge (at $y=0$ ), and the edge states at the other side of the sample have at least 0.85 of their weight fraction above $y=30 \frac{3 \sqrt{3} a}{4}$ (for the armchair edge strip, we take the limits for this procedure to be $x<30 \frac{a}{4 \sqrt{3}}$ and $x>30 \frac{3 a}{4}$, and consider 0.7 of the total wave-function weight rather than 0.85 ). While the criterion we use to identify surface states is arbitrary, we can provide further indication of the validity of this procedure, by plotting the wave functions in real space, and extracting a decay length. We show in Fig. 4 the wave functions of all states we identify as surface states, for the system parameters of Fig. 3(e). We have also fitted these surface states to a wave function profile $|\psi(x)|^{2}$ $=A e^{-x / d} \sin (k x+\phi)^{2}$ and extracted a decay length for each surface state. In Fig. 5 we plot the decay length vs the energy of the surface states, where it can be seen that as we approach the bulk band edges, the decay length diverges. 

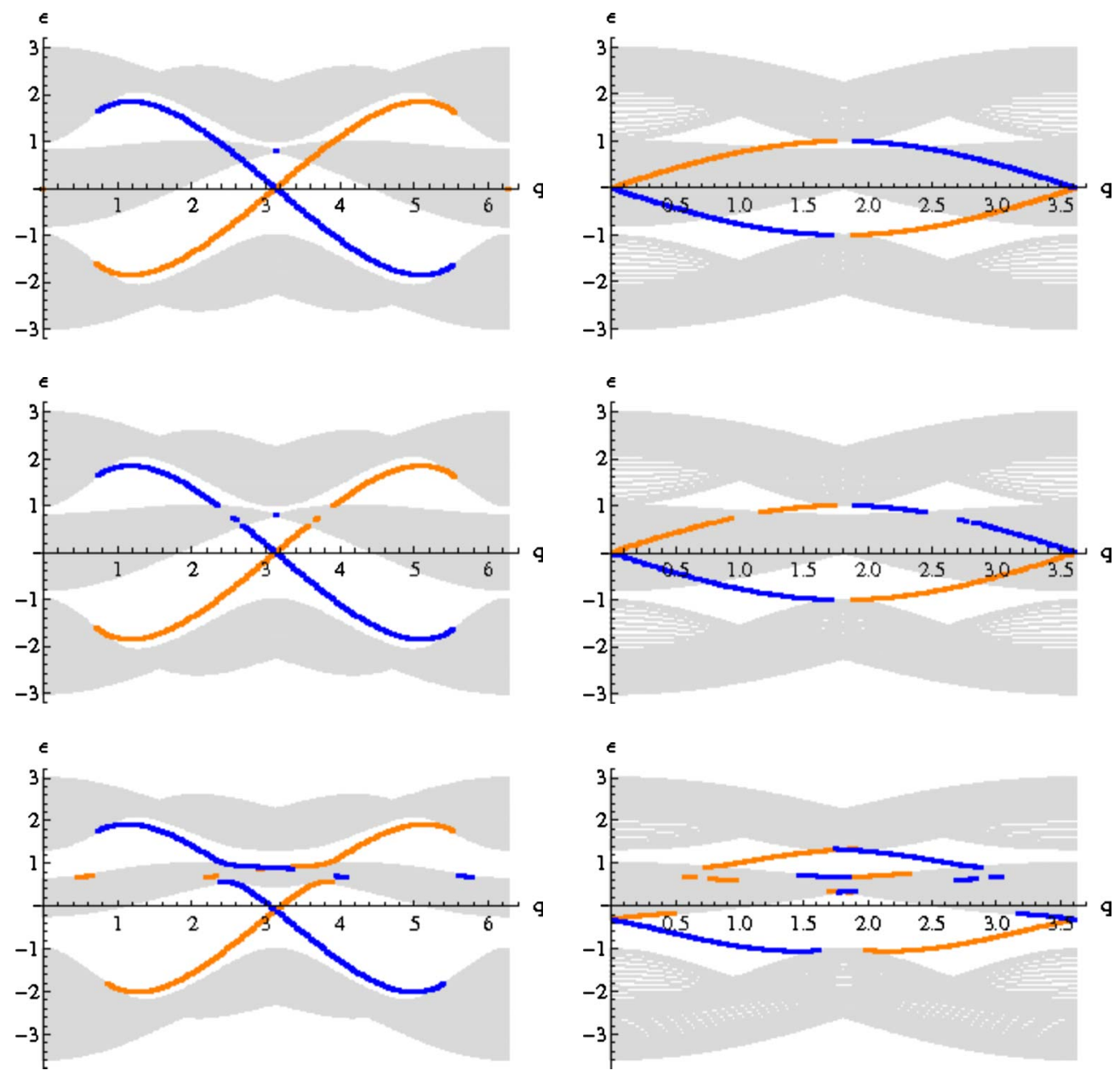

FIG. 3. (Color online) Spectrum of the modified Kane-Mele model in Eq. (1), with a metallic (triangular) band added (Fig. 2). Here we use the parameters $t_{1}=1, \lambda=0.5, t_{2}=0.2, h=0.4$, and vary $t_{3}$. We show spectra for both [(a), (c), and (e)] zigzag and [(b), (d), and (f)] armchair edge strips, with mixing parameter values $t_{3}=0,0.03$, and 0.3 . The strips are finite in one direction, and periodic in the other, so the lattice is wrapped around a cylinder. The edge-state branches on different sides of the sample are denoted by thick lines, dark (blue), and light (orange), respectively. All bulk states are colored light gray. Helical edge-state branches can still appear (at least when the Fermi energy is shifted), as can be easily seen by counting the (odd) number of surface-state branches on one side of the strip crossing $E=0$ between $q=0$ and the BZ midpoint ( $q=\pi$ for zigzag and $q=\pi / \sqrt{3}$ for armchair).

\section{A. Weak hybridization}

In Figs. 3(c) and 3(d), we plot the spectrum of bulk and edge state for an armchair and zigzag strip with weak hybridization, $t_{3}=0.03$. Comparing with the decoupled spectrum in Figs. 3(a) and 3(b), the numerical results clearly show that essentially the same helical surface states appear in our model without a bulk gap present-the helical surface states are only weakly perturbed. Only in those areas where the helical surface states overlap in both energy and momentum with bulk states, do we see any appreciable change. Since different momenta cannot mix in the absence of disorder, it is not surprising the surface states are unaffected if they do not overlap in both energy and momentum.

\section{B. Strong hybridization}

We consider also strong coupling $\left(t_{3}=0.3\right)$ between the honeycomb and triangular subsystems. In the spectra, shown in Figs. 3(e) and 3(f), the surface-state branches have been pushed out of those regions where the bulk states reside, away from their original position, toward those regions of the momentum-energy diagram where bulk states are absent. This results in a doubling of the number of surface-state branches for a single momentum, as can be seen in Fig. 3(e) in the range $2.4<q<3.2$. One branch spans the region below the metallic density of states, and another above.

It is noteworthy that for other parameter choices in this model, one can also find instances where the number of comoving surface states changes upon bulk-edge coupling but still with an odd number of surface branches on each side of the strip (in Appendix B, an example is given where the number of surface states changes from 1 to 3 - still retaining the odd number of branches).

Our numerical results suggest a tendency of surface states to be pushed away from overlapping in (surface-projected) momentum and energy with parasitic bulk states originating from a nontopological band. Not only do they seem to persist, at some momenta they seem to multiply. Motivated by 


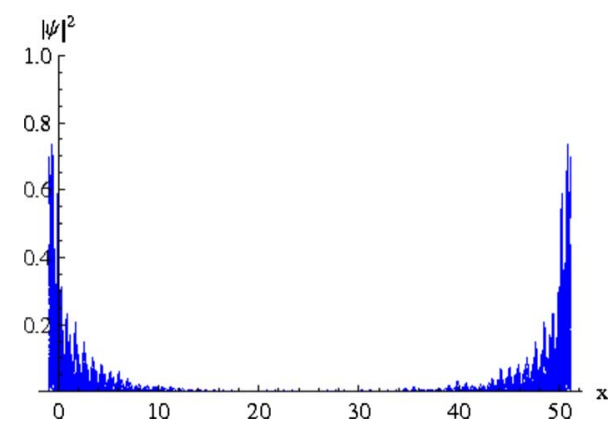

FIG. 4. (Color online) The wave-function density $|\psi|^{2}$ of all 191 eigenstates identified as surface states in Fig. 3(e). The graph shows all wave-function profiles superposed (the point here is to demonstrate that they all decay exponentially into the bulk) as a function of the coordinate $x$ perpendicular to the system boundary. All wavefunction curves are plotted with the same (blue) color.

this observation, we turn next to an analytic treatment of the mixing between surface states and bulk states.

\section{FANO MODEL APPLIED TO EDGE STATES-BULK MIXING}

Our numerical results confirm that edge states have remarkable resilience. Even in the absence of a band gap in the material, precise edge states form in empty spaces in the projected energy-momentum state diagrams. Let us now approach the problem in its idealized form analytically. For this purpose we write a model consisting of a surface-state branch and a decoupled bulk metallic band, and then allow them to mix. This most certainly describes the specific model we have discussed here since an effective low-energy continuum theory would encompass precisely these elements. ${ }^{36}$ We label the transverse momentum by $\mathbf{q}$, and the momentum in the direction perpendicular to the wall by $k$. In the clean limit, the bulk and surface states are mixed by hopping matrix elements which preserve $\mathbf{q}$, and therefore states with different $\mathbf{q}$ do not mix. This allows us to treat individual edge states separately.

The model outlined above takes the form of the wellknown Fano model. ${ }^{35}$ Making use of the path-integral formulation, the action for the model is

$$
\begin{aligned}
S_{1}= & \frac{1}{\beta} \sum_{n} \int_{\mathbf{q}}\left\{\psi^{\dagger} \psi\left[i \omega_{n}-\epsilon(\mathbf{q})\right]+\int_{k} \chi^{\dagger} \chi\left[i \omega_{n}-E(k, \mathbf{q})\right]\right. \\
& \left.+\int_{k}\left[\psi^{\dagger} \chi g(k, \mathbf{q})+\text { H.c. }\right]\right\},
\end{aligned}
$$

where the fermionic Matsubara frequencies are $\omega_{n}=\pi \frac{2 n+1}{\beta}$, and the surface and bulk states are denoted by the Grassmann fields $\psi=\psi\left(i \omega_{n}, \mathbf{q}\right)$ and $\chi=\chi\left(i \omega_{n}, k, \mathbf{q}\right)$, respectively, their dependence on momentum and Matsubara frequency suppressed for the sake of brevity. The surface and bulk state energies are $\epsilon(\mathbf{q})$ and $E(k, \mathbf{q})$, respectively, and the coupling between them is $g(k, \mathbf{q})$. The exact solution of the Fano model $^{37}$ can be most easily achieved by integrating out the bulk degrees of freedom. Since the action is quadratic, this

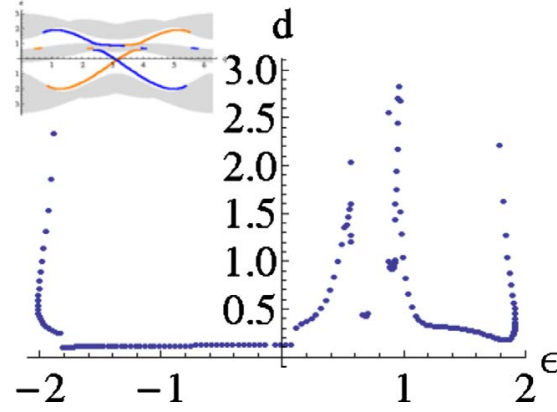

FIG. 5. (Color online) Plot of the surface-state decay length vs the energy of the surface state. We use the same parameters as in Fig. 3(e) (shown in the inset). The energy range $0.6<\epsilon<1$ is where the metallic bulk band appears, and hence a surface-state branch is absent. At all ends of a surface-state branch, where a bulk band appears, a divergence in the decay length is evident. In all cases, the decay lengths are no longer than 3 , in a system of size $\sim 50$. The multivaluedness at the low- and high-energy extremes is due to the surface states curving a bit up in energy as one approaches the original topological insulator bands, as is shown in the inset.

can be done exactly, resulting in an effective action for the surface-state degrees of freedom $(\psi)$ alone,

$$
S_{2}=\frac{1}{\beta} \sum_{n} \int_{\mathbf{q}} \psi^{\dagger} \psi\left[i \omega_{n}-\epsilon(\mathbf{q})-\int_{k} \frac{|g(k, \mathbf{q})|^{2}}{i \omega_{n}-E(k, \mathbf{q})}\right] .
$$

This action reveals the fate of the spectrum of the surface states. The retarded Green's function extracted from the effective action above is

$$
G_{r e t}^{-1}=\left[\omega-\epsilon(\mathbf{q})-\int_{k} \frac{|g(k, \mathbf{q})|^{2}}{\omega-E(k, \mathbf{q})+i \delta}\right] .
$$

From which the spectral function $A(\mathbf{q}, \omega)=$ $-2 \operatorname{Im}\left[G_{r e t}(\mathbf{q}, \omega)\right]$ can be extracted.

The most important feature of the spectral function $A(\mathbf{q}, \omega)$ is that the original surface states, when their energy is within the bandwidth of the bulk, acquire a lifetime, which is roughly $\tau \sim \frac{1}{|g|^{2} \nu\left(E_{F}\right)}$ [where $\nu(E)$ is the density of states and $E_{F}$ the Fermi energy]. This lifetime describes the typical time scale in which the edge probability density leaks into the bulk states. In addition, new delta functions appear at energies outside the bandwidth of the bulk metallic band both above and below. We illustrate this by considering a constant coupling $g$, and a uniform density of states $\nu\left(E_{F}\right)$, in a bulk band with energies $E_{1}<E<E_{2}$. The spectral function is

$$
A(\omega)=\frac{-2 \operatorname{Im}(\Sigma)}{\left\{\omega-\epsilon-\frac{\lambda}{2} \log \left[\frac{\left(\omega-E_{1}\right)^{2}}{\left(E_{2}-\omega\right)^{2}}\right]\right\}^{2}+\operatorname{Im}(\Sigma)^{2}},
$$

where $\lambda=|g|^{2} \nu\left(E_{F}\right)$ and $\operatorname{Im}(\Sigma)=-\pi \lambda$ for $E_{1}<\omega<E_{2}$, and zero if $\omega$ lies outside the bandwidth of the bulk band, thus producing the aforementioned delta functions. Figure 6 shows the spectral function for particular values of the parameters. If the original surface-state energy lies outside the bulk bandwidth, it remains as a delta function outside the bandwidth, slightly shifted from the original surface-state en- 


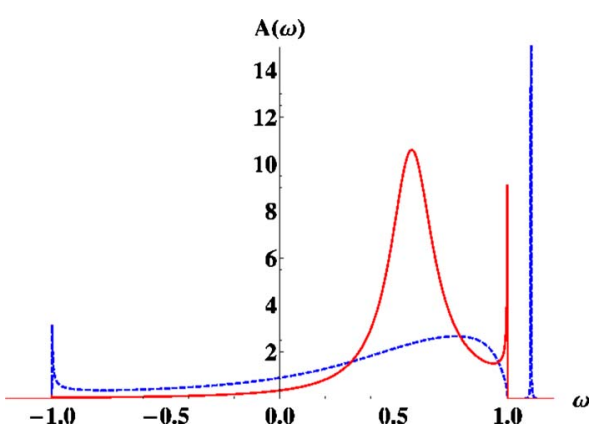

FIG. 6. (Color online) Typical spectral weight function of the Fano model. We take the spectral weight in Eq. (5), with parameters $E_{1}=-1, E_{2}=+1$, the surface-state energy $\epsilon=0.5$ (within the bandwidth). For $\omega$ values outside the bulk bandwidth we take $\lambda=10^{-4}$ to approximate the delta function outside the bandwidth. For the continuous (red) curve we take $|g|^{2} \nu\left(E_{F}\right)=0.04$, and for the dashed (blue) curve we take $|g|^{2} \nu\left(E_{F}\right)=0.2$. At the lower coupling value (continuous curve), the widened peak near $\omega=0.5$ is the remnant of the original surface state, and the modest weight in one delta function already appears very near the band edge (the weight of the other delta function is too small to discern). For the stronger coupling value (dashed line) traces of the surface state are largely obscured in the bulk bandwidth but both the delta functions outside the bandwidth appear prominently.

ergy, and with most of its spectral weight retained. In addition, a new delta-function peak will appear on the other side (in energy) of the bulk band, with a small spectral weight, and some spectral weight will appear within the bulk bandwidth.

These features of the Fano model depend only weakly on the details of the density of states, as can be demonstrated by considering a more realistic bulk band dispersion $E(k)=-\mu$ $-2 t \cos (k)$. Using the dimensionless variable $u \equiv(\omega+\mu) / 2 t$, the integral for the self-energy can be solved analytically, yielding $\operatorname{Re}(\Sigma)=\frac{g^{2}}{t} \frac{\operatorname{sgn}(u)}{\sqrt{u^{2}-1}}$ and $\operatorname{Im}(\Sigma)=0$ when $|u|>1$, and $\operatorname{Re}(\Sigma)=0$ and $\operatorname{Im}(\Sigma)=-\frac{g^{2}}{t \sqrt{1-u^{2}}}$ when $|u|<1$. The resulting spectral function is qualitatively no different than that of Fig. 6, which corresponds to Eq. (5).

The remarkable structure the Fano model implies can be further intuitively understood by considering an impurity state $(d)$ coupled to a flat band of bulk states $\left(f_{n}\right)$ at zero energy, $\mathcal{H}=\epsilon d^{\dagger} d+g \sum_{n}\left[f_{n}^{\dagger} d+\right.$ H.c. $]$ (the coupling $g$ is taken constant without loss of generality), with $n=1 \cdots N$. The characteristic polynomial of the Hamiltonian matrix $\operatorname{Det}(E$ $-\mathcal{H})=(-1)^{N-1} E^{N-1}\left(E^{2}-\epsilon E-N g^{2}\right)$, has $N-1$ zero modes left from the original $N$ flat band states, and two roots at $E$ $=\epsilon / 2 \pm \sqrt{\epsilon^{2} / 4+N g^{2}}$, outside the flat band, which, assuming $N g^{2} \gg \epsilon^{2}$, become $E \approx \pm \sqrt{N} g+\epsilon / 2$. As in the Fano model above, exact energy eigenstates appear above and below the band. While this is an extremely artificial example (flat band states being most sensitive to coupling to other states), it demonstrates how the salient features of the Fano model can be understood-the impurity state "hijacks" one effective mode from the band, and mixes with it, producing two eigenstates with energies outside the band, which correspond to reduced-weight delta functions in the spectral function for $\hat{d}$. Similarly in the general case, the appearance of the second pole in the spectral weight implies that one effective bulk state is hijacked while the effect on most of the band will be minute.

\section{A. Fano model implications}

We can interpret the numerical results of Sec. II in terms of the salient features of the Fano model as follows. The Fano model tells us that for those edge-parallel momenta $\mathbf{q}$ where the surface states and bulk states do not overlap in energy, the surface states are only slightly shifted in energy. This is demonstrated in those regions in Fig. 3 where the original surface states did not overlap with the bulk states. On the other hand, if the surface states overlap in energy with the bulk state energies, the surface states are exiled from the bulk bandwidth, and form states at (very different) lower and higher energies, in addition to leaving ghost surface resonances overlapping with the bulk states, close to where they originated. The exiled states are still surface states, mixed with a superposition of bulk states localized at the surface, since any eigenstate existing in a bulk gap in energy-momentum space, must be an evanescent wave into the bulk (below we will use this fact to derive the penetration

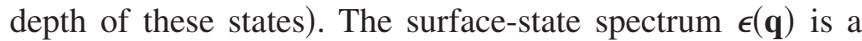
continuous function of $\mathbf{q}$, and assuming the coupling $g(k, \mathbf{q})$ is also a continuous function of momentum, the exiled surface states will also form a $\mathbf{q}$-continuous energy branch. The helical nature of the surface states will also be preserved in the new exiled surface states: it will correspond to an odd number of Dirac cones in 3D (and an odd number of Kramer's pairs of bands in 2D). The exiled states are evident in the numerics in Figs. 3(e) and 3(f), in particular, in Fig. 3(e) in the range $2.4<q<3$.2. Indeed, the exiled states retain the surface-state branch continuity, as well as their helical nature. The ghost surface resonances are not pure surface states, and so are not distinguished in the spectra in Fig. 3. In order to identify the "ghost state" signatures in our numerical, we probe the explicit spectral weight function of our model in the next section.

Experimentally, we expect that on different facets of the crystal the two scenarios could be realized, and so if a helical surface state appears on one facet of the metal, crossing the Fermi energy, but not on the other facets, it may simply be significantly shifted in energy, and can in principle still be observed outside the energy range of the bulk band.

In topological insulators, the surface-state branches should be anchored at one end in the valence band, and at the other end at the conduction band. In finite size TI systems, the connection of the topological surface state and the bulk bands is actually avoided only by a finite-size minigap, presumably of order $1 / L$, where $L$ is the system size (this can be seen explicitly in Fig. 1 of Ref. 1). Likewise in our system, the exiled states come infinitesimally close to the bulk band edge-only a finite-size limited gap of order $1 / L$ should appear between the surface branch and the bulk band. The Fano model predicts the exiled states come infinitesimally close to the band edge. Therefore, the helical surface-state "connecting effect" is no different from that in proper TI band structures. 
In order to learn something about the exiled states, we can use the fact that they appear in band-gap regions to estimate their skin depth. Assuming a surface state is at an energy $\Omega$ close to only one band, and far away from all other bands, it can be thought of as an evanescent wave at the interface between the vacuum and a solid with the dispersion relation of the bulk $E \approx \Delta+\frac{k^{2}}{2 m}$, where $\Delta>\Omega>0$ is the energy of the bottom of the band. This dispersion relation translates into a wave equation in space time

$$
i \partial_{t} \psi \approx\left(\Delta-\frac{1}{2 m} \partial_{x}^{2}\right) \psi,
$$

for $x>0$ (in the solid) while $\Delta \rightarrow 0$ (and perhaps the mass changes) in the vacuum $(x<0)$. For $x>0$, the wave function should be of the form $\psi \sim e^{-x / d-i \epsilon t}$ with some penetration depth $d$, having a positive real part. Substituting this in the bulk wave equation, we find

$$
d=[2 m(\Delta-\Omega)]^{-1 / 2} .
$$

As the surface state gets closer to the band edge $\Omega \rightarrow \Delta$, the penetration depth diverges, as seen in Fig. 5.

While the rough analysis above strongly suggest that the surface states' lines appearing in the band gaps can only represent exponentially decaying eigenstates, a more thorough analysis is possible. Starting from Eq. (2), we consider one parallel momentum point $\mathbf{q}$, which for the sake of brevity, we will omit it from our notation. As explained above, there appear two eigenstates in the gaps above and below the bandwidth of the bulk band. Choosing one of them (with energy $\Omega$ ), the (surface) eigenstate in the hybridized system must be some superposition of the original surface state and the bulk states $|\phi\rangle=\alpha|\psi\rangle+\int_{k} \beta_{k}|\chi\rangle$ The spatial wave function is found as

$$
\phi(x)=\langle x \mid \phi\rangle=\alpha\langle x \mid \psi\rangle+\int_{k} \beta_{k}\langle x \mid \chi\rangle \sim \alpha e^{-\lambda x}+\int_{k} \beta_{k} e^{i k x} .
$$

The first term above is clearly exponentially decaying-this is the part coming directly from the original surface-state wave function. The second term, however, may at first sight have a very different functional shape. It is this function $f(x) \equiv \int_{k} \beta_{k} e^{i k x}$ we wish to calculate. The details of deriving this quantity are a bit involved, and we leave them for Appendix $\mathrm{C}$ and simply state the result here

$$
f(x) \sim \int_{k} e^{-i k x} \frac{g(k)}{\Omega-E_{k}},
$$

which for the simple example of $g=$ const and $\Omega-E_{k}=\Delta$ $+2 t[\cos (k)+1]$ can be shown to result in

$$
f(x) \sim e^{-i \pi x} e^{-x \ln \left\{1+(\Delta / 2 t)+\sqrt{[1+(\Delta / 2 t)]^{2}-1}\right\}},
$$

clearly an exponentially decaying function.

\section{B. Numerical evidence for the surface resonance}

In this section we will present numerical evidence for the ghost state surface resonance in the model described in Sec.

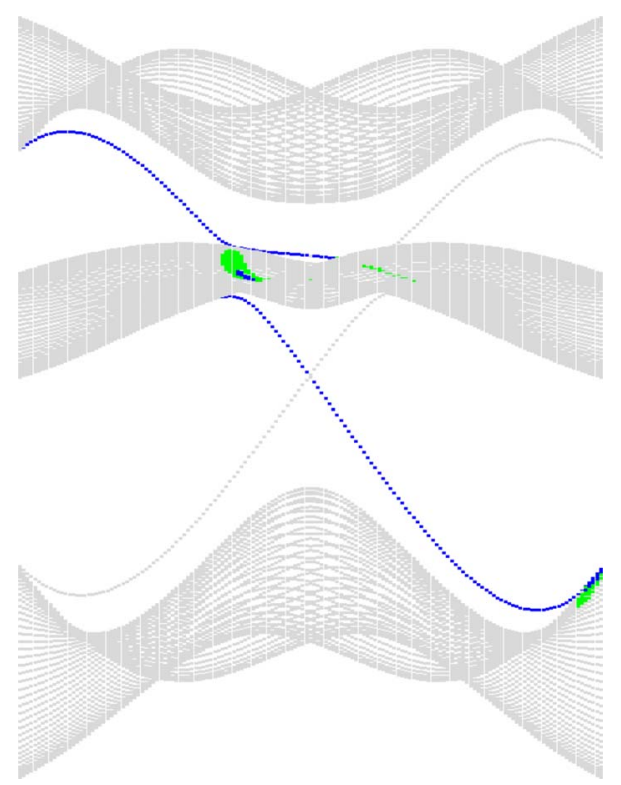

FIG. 7. (Color online) Numerical calculation of the surface-state spectral function. We take the surface-state eigenstates in the decoupled case $\left[\psi_{0}(\mathbf{q})\right]$ and find their distribution among the eigenstates of the coupled system $\phi_{\omega}(\mathbf{q})$ by calculating the overlaps $A(\mathbf{q}, \omega)$ $=\left|\left\langle\psi_{0}(\mathbf{q}) \mid \phi_{\omega}(\mathbf{q})\right\rangle\right|^{2}$. This is expected to approximate Eq. (5). We use the same parameters as the strongly hybridized zigzag edge strip in Fig. 3(e), and the overall spectrum is manifestly identical in the two images. We plot the surface-state spectral weight function versus transverse momentum (horizontal axis) and energy (vertical axis). Dark (blue) points have $A(\omega)>0.06$, intermediate gray (green) points have $0.06>A(\omega)>0.02$, and all points with $0.02>A(\omega)$ are very light gray. The exiled surface-state branches are manifest, and the ghost spectral resonance is identifiable as a faint peak overlapping with the bulk band, between the two exile branches. In addition, the lower spectral weight points map out the shadow of the full bulk spectrum.

II. First, we calculate explicitly the surface-state spectral weight function [Eq. (5)] described above in the Fano model analysis. This is done by calculating a probability distribution to find the original surface-state eigenstates at a given energy and momentum in the new, coupled spectrum. We take the surface-state eigenstates $\psi_{0}(\mathbf{q})$ in the decoupled case, and calculate their overlap with the various eigenstates of the coupled system, squared. In particular, if we denote by $\phi_{\omega}(\mathbf{q})$ all the eigenstates of the coupled system, with energies $\omega$, the spectral weight is $A(\mathbf{q}, \omega)=\left|\left\langle\psi_{0}(\mathbf{q}) \mid \phi_{\omega}(\mathbf{q})\right\rangle\right|^{2}$. The results of our numerics, for the surface-state branch along one edge of the sample in the strong coupling case $\left(t_{3}\right.$ $=0.3$ ), are shown in Fig. 7. A faint but discernible diffuse peak is seen to overlap with the bulk states in between the upper and lower exiled surface-state lines, thus confirming the predictions from the Fano model analysis.

The spectral weight function [Eq. (5)] is not necessarily what ARPES or scanning tunnel microscope will measure. In order to give a clear experimental signature that can be measured, we perform one additional numerical analysis. We take the density profile of each eigenstate $\phi_{\omega}(\mathbf{q})$, and convolve it with a weight factor $f(y)=e^{-y / 4 a}$, to yield the total weight $\mathcal{J}(\omega, \mathbf{q})=\left|\left\langle\phi_{\omega}(\mathbf{q})|f(y)| \phi_{\omega}(\mathbf{q})\right\rangle\right|^{2}$. The decaying expo- 

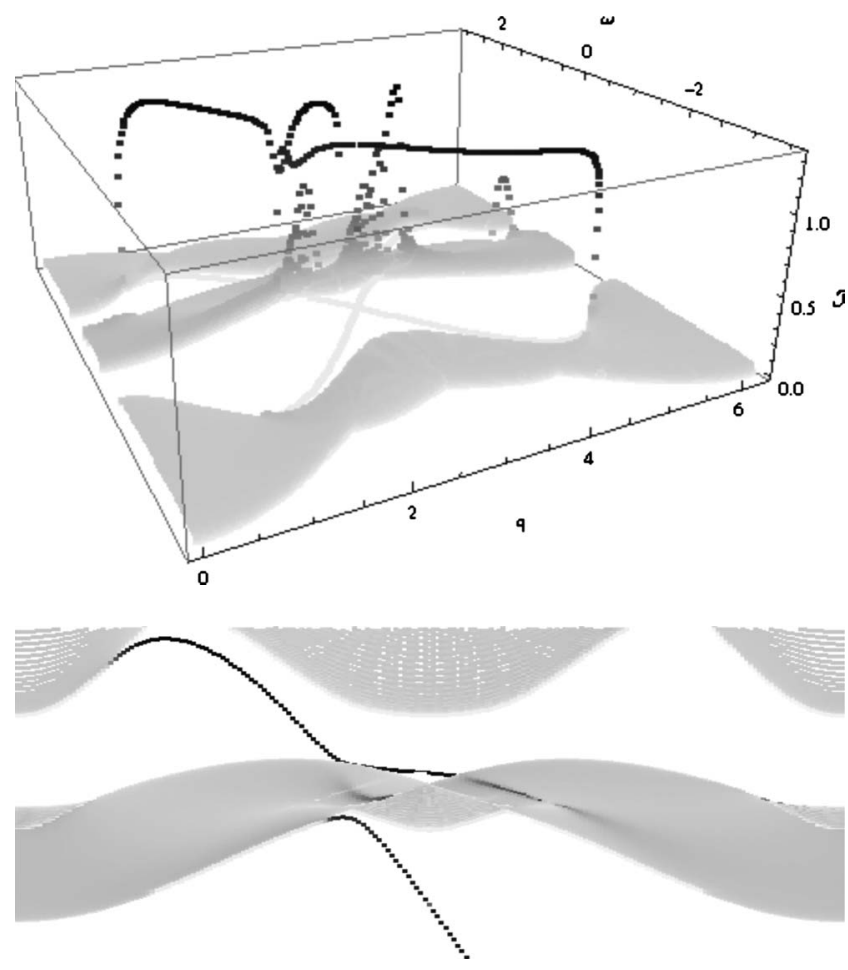

FIG. 8. The ghost surface resonance observed in surface probes. The quantity $\mathcal{J}$ measures the weight of each eigenstate $\phi_{\omega}$ convolved with an exponentially decaying weight factor, mimicking the penetration depth of the experimental surface probes $\mathcal{J}(\omega, \mathbf{q})$ $=\left|\left\langle\phi_{\omega}(\mathbf{q})|f(y)| \phi_{\omega}(\mathbf{q})\right\rangle\right|^{2}$. Here we plot $\mathcal{J}$ versus transverse momentum $\mathbf{q}$ and energy $\omega$, both in (a) 3D and (b) 2D plots. The grayscale color scheme is such that higher values are colored dark gray. We find significant values of $\mathcal{J}$ only for the surface states and the surface resonances, which are manifestly positioned in between the exiled surface states [most clearly in the 2D plot (b)]. In addition, the very light gray points map out the bulk states.

nential mimics the finite penetration depth surface probes can achieve. Advantageously, the calculation of $\mathcal{J}$ is unbiased by the "band archeology" in calculating $A(\mathbf{q}, \omega)$, which required comparison with the eigenstates of the decoupled system. We plot $\mathcal{J}(\omega, b f q)$ versus $\omega$ and $\mathbf{q}$ in Fig. 8. Those eigenstates with a significant part localized at the surface should have a sizable value of $\mathcal{J}$. Indeed, we see in Fig. 8 that between the exiled surface states the signature of the ghost surface resonance appears. In conclusion, careful analysis of our numerical results shows that surface-state probes could identify the ghost surface resonance.

\section{DISORDERED HELICAL METAL WITHIN THE FANO MODEL}

Going beyond the clean limit, we now consider edge-bulk hybridization in the presence of disorder. A random disorder potential scatters surface states into other surface states, as well as into the bulk. For simplicity we ignore, however, disorder scattering between bulk states, concentrating only on the fate of the surface states. Within the weak-disorder approximation $^{38}$ we will see that the Fano picture by and large still applies.
Disorder scattering can easily be quantified by slightly modifying Eq. (2),

$$
\begin{aligned}
S_{3}= & \frac{1}{\beta} \sum_{n} \int_{\mathbf{q}}\left\{\psi^{\dagger} \psi\left[i \omega_{n}-\epsilon(\mathbf{q})\right]+\int_{k} \chi^{\dagger} \chi\left[i \omega_{n}-E(k, \mathbf{q})\right]\right. \\
& \left.+\int_{k, \mathbf{q}^{\prime}}\left[\chi^{\dagger} \psi^{\prime} g\left(k, \mathbf{q}, \mathbf{q}^{\prime}\right)^{*}+\text { H.c. }\right]+\int_{\mathbf{q}^{\prime}} \psi^{\dagger} \psi^{\prime} V\left(\mathbf{q}, \mathbf{q}^{\prime}\right)\right\},
\end{aligned}
$$

where we use the shorthand notation $\psi^{\prime} \equiv \psi\left(i \omega_{n}, \mathbf{q}^{\prime}\right)$ (and similarly for $\psi^{\prime \prime}$ later on). The coupling term $g\left(k, \mathbf{q}, \mathbf{q}^{\prime}\right)$ no longer conserves transverse momentum and is determined by the overlap of the surface $(\widetilde{\psi})$ and bulk $(\widetilde{\chi})$ state wave functions represented by the various fields weighted by the random potential, $g\left(k, \mathbf{q}, \mathbf{q}^{\prime}\right)=\int_{\mathbf{r}} \widetilde{\psi}_{\mathbf{q}^{\prime}}(\mathbf{r})^{*} U(\mathbf{r}) \widetilde{\chi}_{k, \mathbf{q}}(\mathbf{r})$. Integrating out the bulk fermions, we are left with the effective action,

$$
\begin{aligned}
S_{4}= & \frac{1}{\beta} \sum_{n}\left[\int_{\mathbf{q}} \psi^{\dagger} \psi\left[i \omega_{n}-\epsilon(\mathbf{q})\right]+\int_{\mathbf{q}, \mathbf{q}^{\prime}} \psi_{\mathbf{q}}^{\dagger} \psi_{\mathbf{q}^{\prime}} V\left(\mathbf{q}, \mathbf{q}^{\prime}\right)\right. \\
& \left.-\int_{k, \mathbf{q}, \mathbf{q}^{\prime}, \mathbf{q}^{\prime \prime}}\left(\psi^{\prime}\right)^{\dagger} \psi^{\prime \prime} \frac{g\left(k, \mathbf{q}, \mathbf{q}^{\prime}\right)^{*} g\left(k, \mathbf{q}, \mathbf{q}^{\prime \prime}\right)}{i \omega_{n}-E(k, \mathbf{q})}\right] .
\end{aligned}
$$

Next we average over disorder, assuming the disorder has a Gaussian distribution $\overline{U(\mathbf{r}) U\left(\mathbf{r}^{\prime}\right)}=C\left(\mathbf{r}-\mathbf{r}^{\prime}\right)=\widetilde{u}^{2} \delta\left(\mathbf{r}-\mathbf{r}^{\prime}\right)$, and surface and bulk wave functions $\tilde{\psi} \sim e^{i \mathbf{q} \cdot \mathbf{R}-|x| / d}$ and $\tilde{\chi}$ $\sim e^{i \mathbf{q} \cdot \mathbf{R}+i k x}$ (where the edge-parallel and perpendicular coordinates have been separated as $\mathbf{r}=(x, \mathbf{R})$, and $d$ is the skin depth of the surface states). Finally, expanding in the limit of weak disorder we find the leading contribution from disorder is a term quadratic in $\psi$ (higher order terms in $\psi$ are weaker in this limit)

$$
S_{5}=\frac{1}{\beta} \sum_{n} \int_{\mathbf{q}} \psi^{\dagger} \psi\left\{\left[i \omega_{n}-\epsilon(\mathbf{q})\right]-\int_{k, \mathbf{q}^{\prime}} \frac{u^{2}}{i \omega_{n}-E\left(k, \mathbf{q}^{\prime}\right)}\right\},
$$

where $u$ differs from $\tilde{u}$ by some numerical constant. It is important to note that this leading term originates solely from the bulk-surface-state scattering, and can also be derived by simply taking the disorder mean of the action in Eq. (12). This gives a Green's function nearly identical to that of Eq. (4) but we notice that while transverse momentum is now once again a good quantum number, disorder, even after averaging, couples all bulk states to any one of the surface states. Therefore, the separation in energy and momentum picture no longer holds here, and any surface state that does not exist in a full energy band gap will suffer the effects of a Fano mode impurity state in the energy bandwidth of the bulk band. From the original surface-state branch, a ghost feature will remain in the spectral function, and exiled surface-state delta functions appear outside the bulk bandwidth. When disorder is very weak, the ghost surface states will actually be the most noticeable feature and will simply seem as ordinary surface states with a lifetime to leak into the bulk, determined by the strength of the disorder.

A simple experimental test for our predictions follows from the observation that the scattering strength is propor- 
tional to impurity density: $u^{2} \propto \rho_{\text {imp }}$. In samples where the disorder scattering of surface states into the bulk dominates over other effects (phonon scattering and electron-electron interactions), the surface-state lifetime must be inversely proportional to the impurity density. Very recent work $^{39}$ has already measured a quasiparticle lifetime for the surface states in $\mathrm{Bi}_{2} \mathrm{Se}_{3}$, which is one of the main candidates to be a helical metal as mentioned above, and concluded from the energy dispersion of the surface-state lifetime that disorder scattering seems to be the dominating scattering mechanism. It would be particularly interesting in such experiments to search for the exiled states, which should be above and below the bandwidth of the bulk band. We leave a theoretical study of the nature of the exiled states in disordered helical metals to future work.

The difference between helical surface states and nonhelical surface states becomes paramount when considering disorder. Nonhelical surface states, in the clean limit, consist of an even number of bands that can mix via time-reversal preserving terms. In 2D materials with one-dimensional (1D) surface states, nonmagnetic disorder, of the likes we consider here, can backscatter between two such bands since their spin configurations are nonorthogonal, and therefore strongly localize them (although a strong spin-orbit interaction may mitigate this effect $\left.{ }^{40}\right)$. A helical edge state on the other hand is a chiral 1D conductor, which suffers no backscattering. In 3D materials the surface states are two dimensional, and nonmagnetic disorder would lead to weak localization. ${ }^{41} 3 \mathrm{D}$ helical surface states, however, always exhibit antilocalization of a single Dirac cone. Nonhelical surface states may also be in an antilocalization class due to spin-orbit coupling but unlike the helical states, they are (topologically) smoothly connected to a spin-rotationally symmetric $2 \mathrm{D}$ electron gas (2DEG), which suffers weak localization. The additional protection that helical surface states exhibit against localization effects indicates that the surface-bulk Fano effects will dominate over localization effects, whereas the opposite may be true for nonhelical systems.

\section{CONCLUSIONS}

Topological band insulators are characterized by their surface-state properties. Helical states on the surface of a TI have $o d d$, rather than even, number of either Kramer's pairs of 1D surface channels in a 2D material, or 2D surface metals with a Dirac dispersion in a 3D material. ${ }^{1-3,8,9}$ In our work we demonstrated that even materials that are generically metals retain features of the helical structure of the surface states, and therefore a similar classification can apply to metallic states.

Furthermore, we find that the mixing between surface states and bulk states in metals results in a generic rearrangement of the surface-state spectrum. At energies where surface and bulk states overlap and mix (either when they overlap also in momentum parallel to the surface or due to disorder) the surface states are reduced into surface resonance ghosts, with a diffused spectral function peak centered near the original energy of the surface states. In addition, the bulk-surface mixing produces "exiled surface states" outside the confines of the bulk parasitic metal energy bandwidth. Therefore, the surface-state spectral structure emerging from this mixing contains for each surface momentum $\mathbf{q}$ (where surface states exist) two sharp surface states above and below the parasitic metal bands where there is a bulk gap (for that momentum number, or, when weak disorder is at play, a complete bulk gap), and a diffuse surface resonance overlapping with the metallic density of states. Surface resonances, despite being immersed in the bulk states, can still be identified in ARPES measurements due to the fact that they have very weak $k$ dispersion ${ }^{42}$ (and ideally none), in contrast to bulk states. Following the surface resonance peak for different q's should roughly parallel the sharp surface-state spectrum above and below the band. The location of the sharp exiled states and the diffuse ghost resonance may change for surfaces made of different facets (cuts) of the crystal. For instance, at a particular energy a sharp edge state on one facet may correspond to a mere ghost resonance when we consider another surface.

The evolution of the surface-state spectrum in the hybridized system, as described above, makes it clear that the helical characteristic of the surface states will remain unchanged. The topological insulator has only one Kramer's doublet of surface-state branches: this degeneracy cannot be lifted by the mixing with the bulk band, and therefore it is sufficient to consider a generalized Fano analysis of only a single surface branch with the bulk. In the nontopological case we would always have to consider two surface branches, which may mix due to the hybridization with the bulk. Strikingly, in some cases the number of surface-state branches at each edge can change but the parity of the number of surface bands will remain odd (see Appendix B, where the one surface-state branch gets multiplied to 3 ).

Effects of disorder were only briefly and crudely considered here. Nevertheless we can already indicate intriguing features which may arise. Following the disordered Fano model for the case of a metallic band overlapping in energy but not in momentum, the hybridization will broaden a surface state (say at momentum q) into a ghost but will also produce sharp (surface) energy eigenstates at energies above and below the energy overlap range, so long as there is an empty patch as a function of energy that can support them. An ARPES measurement should be able to observe all these features for metals with an appropriate band structure, where a metallic parasitic band overlaps in one range of energies with topological edge states, but not in momentum, and above or below this energy range bulk gaps exist. Tunneling measurements should also be able to observe the spectral features we describe here. We will explore this situation more closely in future work. A more mundane prediction of the disorder analysis which should be easily accessible in experiment is the quasiparticle lifetime decrease due to increasing disorder.

Topological insulators (and topological phases in general) are characterized by a topological invariant that assumes only quantized values, and is thus robust to infinitesimal deformations to the model. The presence of edge states is deeply connected to topological order, and the presence of helical edge states in the metallic models we present here would suggest some sort of topological order may exist. 
However, conventional understanding of topological phases depends crucially on having a robust global gap in the system. The "helical metal" phase we consider here is gapless, though in a clean system local gaps in the BZ may appear. It is therefore unclear whether one can define a topological invariant in general. However, one incarnation of the topological invariant for the topological insulators (in both $d$ $=2,3$ ), involves Bloch states only at time-reversal invariant momenta in the BZ. ${ }^{8}$ If there is a local gap at these points in the BZ, the same topological invariants are still well defined. In the helical metal phases we introduce here, each of these points can either be locally gapped or not. It is also unclear what could be a topological invariant for the helical metal in the presence of disorder. Going forward, the question whether a topological invariant exists for the systems we introduce here is perhaps the most enticing, and we leave its determination to future work.

The abundance of materials exhibiting topological properties suggests that there must be many materials which are helical metals - metals with an odd number of chiral surface states at some energy ranges for each facet. Such materials can presumably be found in the vicinity of topological insulators and vice versa, which is supported by the materials observed so far, $\mathrm{Sb}, \mathrm{Bi}_{1-x} \mathrm{Sb}_{x}$, and $\mathrm{Bi}_{2} \mathrm{Se}_{3}$. The spectral effects which we explore here should be accessible in all of these materials and provides another challenging system where interaction and disorder may have important and interesting effects.

\section{ACKNOWLEDGMENTS}

It is a pleasure to acknowledge useful discussions with P. A. Lee, O. Motrunich, Z. Hasan, and D. Hsieh. D.L.B. is grateful for support by the Sherman Fairchild Foundation. G.R. is grateful for the generous support of the Packard Foundation, the Sloan Foundation, and the FENA Focus Center, one of six research centers funded under the Focus Center Research Program (FCRP), a Semiconductor Research Corporation entity.

\section{APPENDIX A: ALTERNATIVE CONSTRUCTION OF A HELICAL METAL MODEL}

Here we explore an example of an alternative construction of a helical metal model, without introducing new degrees of freedom. Both constructions were briefly outlined in Sec. II. We start with the Kane-Mele model, ${ }^{1,3}$ and add second neighbor hopping $\widetilde{t}_{2}$ (which involves hopping only on the same sublattice). This realizes the construction leading to a momentum-dependent chemical potential. We adjust the second neighbor hopping and overall chemical potential to be strong enough to close the bulk gap while having a near zero value near those momenta at which the surface states appear, on the zigzag edge surface of the honeycomb lattice. We explore both this edge as well as the armchair edge. Our model is most simply and succinctly written as
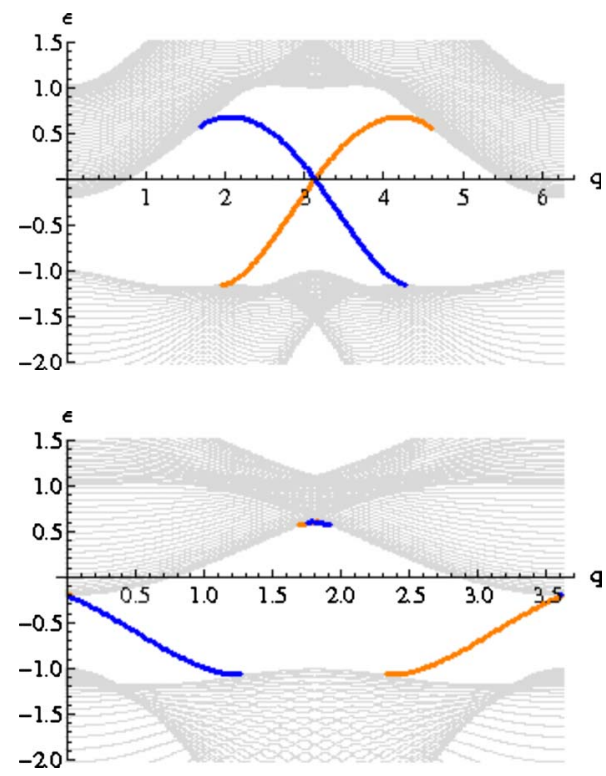

FIG. 9. (Color online) All pictorial conventions are exactly the same as in Fig. 3, including the system size, and the criteria for identifying surface states. Here we use parameters $\lambda=0.3, \mu=0.8$, $t_{1}=1$, and $\tilde{t}_{2}=0.4$. We show the zigzag spectrum in (a) and the armchair edge spectrum in (b).

$$
\begin{aligned}
\mathcal{H}= & -t_{1} \sum_{\langle i j\rangle \alpha}\left[a_{i \alpha}^{\dagger} b_{j \alpha}+\text { H.c. }\right]-\mu \sum_{j \alpha} a_{j \alpha}^{\dagger} a_{j \alpha}+(a \rightarrow b) \\
& +\sum_{\langle\langle i j\rangle\rangle \alpha \beta}\left[a_{i \alpha}^{\dagger} a_{j \beta}\left(-\tilde{t}_{2} \delta_{\alpha \beta}+i \lambda s_{\alpha \beta}^{z} \nu_{i j}\right)+\text { H.c. }+(a \rightarrow b)\right],
\end{aligned}
$$

where as in Eq. (1), $a, b$ denote the fermion operators on the two sublattices, $i, j$ denote the lattice sites, the spin indices are denoted by $\alpha, \beta$, and $\nu_{i j}$ is as defined in Refs. 1 and 3 (and shown in Fig. 2). Repeating the finite strip numerical diagonalization for both zigzag and armchair edges, we find the results of Figs. 9(a) and 9(b), demonstrating yet again the presence of helical surface states coexisting with a bulk Fermi surface. The surface states in the armchair edge geometry seem as if they have been pushed away from overlapping with the bulk states, reminiscent of the exiling effects we discussed in the main text.

Note that some examples of helical metals may also have surface states on some faces but not on others, as would be the case in the above model if the second-nearest-neighbor hopping were sufficiently strong such that no bulk gap would exist. Generically, such surface states will be unstable to strong disorder, and will be subject to a finite lifetime as described in Sec. IV above.

\section{APPENDIX B: SURFACE-STATE MULTIPLYING}

Another revealing example of the model in Eq. (1) shows the helical nature of the surface states being preserved despite significant changes in the band structure. The parameters used here in this example are $\lambda=0.08, t_{2}=-1, t_{1}=1, h$ $=2$, and $t_{3}=0.5$, and we, once again, repeat the same numerical calculation on both the zigzag and armchair edged strip 

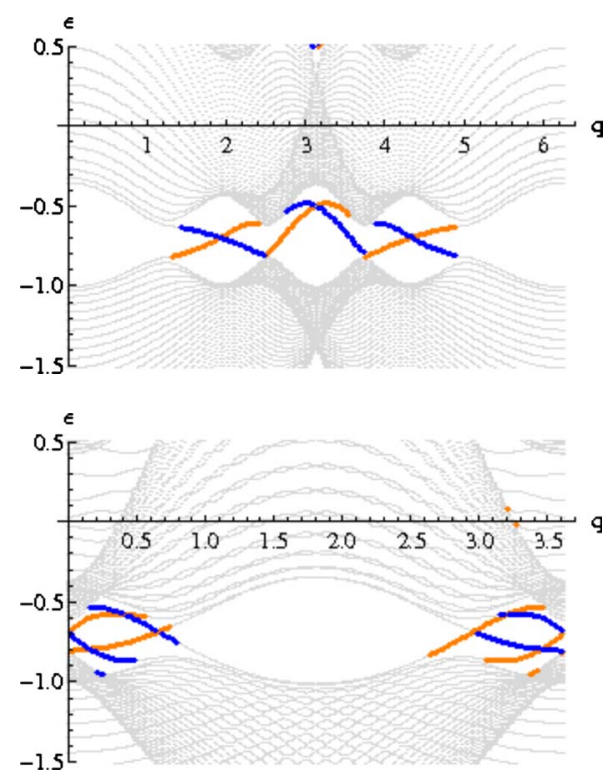

FIG. 10. (Color online) All pictorial conventions are exactly the same as in Fig. 3, including the system size, and the criteria for identifying surface states. Here we use parameters $\lambda=0.08, t_{2}=-1$, $t_{1}=1, h=2$, and $t_{3}=0.5$. We show the spectrum of both zigzag and armchair edge strip geometries. The same expulsion of the surface states occurs, as in Fig. 3, and in addition we find the number of surface branches has tripled to three Kramer's pairs on each wall of the strip.

geometries. The various spectra are displayed in Fig. 10 and show that on both edges, the single surface-state branch has multiplied into three copropagating surface-state branches, albeit not crossing the Fermi energy (though that can be changed with the overall chemical potential) so that the total number of helical surface states on one edge has changed from one Kramer's pair of bands to three. There are still an odd number of surface-state branch pairs, which means the system has the same helical classification. This numerical result again confirms the robustness of the helical nature of the surface states in this model, despite strong mixing with an ordinary metallic band. We will leave the exploration of the edge-state multiplication effect to future work.

\section{APPENDIX C: CALCULATION OF THE HYBRIDIZED WAVE FUNCTION FORM}

Our goal is to calculate the quantity $\beta_{k}=\left\langle\chi_{k} \mid \phi\right\rangle$, defined in Sec. III A. We can find this using Green's-function methods. The Green's function of the full hybridized system can be written in its spectral decomposition as

$$
G=\sum_{\lambda} \frac{\left|\phi_{\lambda}\right\rangle\left\langle\phi_{\lambda}\right|}{\omega+i \eta-\Omega_{\lambda}}
$$

where $\Omega_{\lambda}$ are the eigenstate energies. We are interested in a particular (surface) eigenstate, with the isolated energy value of $\Omega_{\lambda}$. Taking a contour integral $C_{0}$ in $\omega$ surrounding only this pole of the Green's function we find

$$
\oint_{C_{0}} \frac{d \omega}{2 \pi i} G=\left|\phi_{\lambda}\right\rangle\left\langle\phi_{\lambda}\right| .
$$

From this point on we will drop the subscript $\lambda$. The quantity we are after can then be calculated from

$$
\beta_{k} \alpha^{*}=\left\langle\chi_{k} \mid \phi\right\rangle\langle\phi \mid \psi\rangle=\oint_{C_{0}} \frac{d \omega}{2 \pi i}\left\langle\chi_{k}|G| \psi\right\rangle .
$$

It is useful to note that the quantity $\langle\psi|G| \psi\rangle$ is precisely the retarded Green's function of Eq. (4), and we will now show that $\left\langle\chi_{k}|G| \psi\right\rangle$ can easily be calculated using $\langle\psi|G| \psi\rangle$. With a Hamiltonian $H_{0}+H_{1}$ corresponding to the action in Eq. (2), with $H_{1}$ being the hybridization term, the full Green's function can be written as

$$
G=\left[G_{0}^{-1}-H_{1}\right]^{-1}=\left[1-G_{0} H_{1}\right]^{-1} G_{0}=\sum_{n=0}^{\infty}\left(G_{0} H_{1}\right)^{n} G_{0},
$$

where $G_{0}^{-1}=\omega+i \delta-H_{0}$, and in the spectral decomposition

$$
G_{0}=\frac{|\psi\rangle\langle\psi|}{\omega+i \delta-\epsilon}+\int_{k} \frac{|\chi\rangle\langle\chi|}{\omega+i \delta-E_{k}}
$$

and

$$
H_{1}=\int_{k} g(k)|\chi\rangle\langle\psi|+\text { H.c. }
$$

Using the facts that $\left\langle\chi\left|G_{0}\right| \psi\right\rangle=0$ and $\langle\chi| G_{0} H_{1}=\frac{g(k)}{\omega+i \delta-E_{k}}\langle\psi|$ we find that

$$
\begin{aligned}
\langle\chi|G| \psi\rangle & =\left\langle\chi\left|\sum_{n=1}^{\infty}\left(G_{0} H_{1}\right)^{n} G_{0}\right| \psi\right\rangle \\
& =\left\langle\chi\left|\left(G_{0} H_{1}\right) \sum_{n=0}^{\infty}\left(G_{0} H_{1}\right)^{n} G_{0}\right| \psi\right\rangle \\
& =\left\langle\chi\left|\left(G_{0} H_{1}\right) G\right| \psi\right\rangle \\
& =\frac{g(k)}{\omega+i \delta-E_{k}}\langle\psi|G| \psi\rangle .
\end{aligned}
$$

Using Eq. (4), we then have

$$
\begin{aligned}
\beta_{k} \alpha^{*} & =\oint_{C_{0}} \frac{d \omega}{2 \pi i}\langle\chi|G| \psi\rangle \\
& =\oint_{C_{0}} \frac{d \omega}{2 \pi i} \frac{g(k)}{\omega+i \delta-E_{k}} \frac{1}{\omega+i \delta-\Sigma(\omega)},
\end{aligned}
$$

where the self-energy $\Sigma(\omega)$ is that appearing in Eq. (4). The contour $C_{0}$ by definition encircles only the pole of the $\langle\psi|G| \psi\rangle$ part, and since we already know this energy $\Omega$ 
appears in the bulk gaps, the poles $E_{k}$ in the integral are outside the contour. Then by the residue theorem

$$
\beta_{k} \alpha^{*}=\frac{g(k)}{\Omega+i \delta-E_{k}} \frac{1}{1-\left.\frac{\partial \Sigma}{\partial \omega}\right|_{\Omega} .}
$$

Since $\alpha$ is a constant, all the momentum dependence comes only from the factor

$$
\beta_{k} \sim \frac{g(k)}{\Omega+i \delta-E_{k}}
$$

and we finally can write

$$
f(x)=\int_{k} e^{-i k x} \beta_{k} \sim \int_{k} e^{-i k x} \frac{g(k)}{\Omega+i \delta-E_{k}},
$$

which is precisely (after $\delta \rightarrow 0$ ) Eq. (9) in the main text. Note that since the energy $\Omega$ is outside the bandwidth of $E_{k}$, the denominator in the integrand has no singularity.
${ }^{1}$ C. L. Kane and E. J. Mele, Phys. Rev. Lett. 95, 226801 (2005).

${ }^{2}$ B. A. Bernevig, T. L. Hughes, and S.-C. Zhang, Science 314, 1757 (2006)

${ }^{3}$ C. L. Kane and E. J. Mele, Phys. Rev. Lett. 95, 146802 (2005).

${ }^{4}$ C. L. Kane and E. J. Mele, Science 314, 1692 (2006).

${ }^{5}$ B. A. Bernevig and S.-C. Zhang, Phys. Rev. Lett. 96, 106802 (2006).

${ }^{6}$ C. Wu, B. A. Bernevig, and S.-C. Zhang, Phys. Rev. Lett. 96, 106401 (2006).

${ }^{7}$ M. Konig, S. Wiedmann, C. Brune, A. Roth, H. Buhmann, L. W. Molenkamp, X.-L. Qi, and S.-C. Zhang, Science 318, 766 (2007).

${ }^{8}$ L. Fu, C. L. Kane, and E. J. Mele, Phys. Rev. Lett. 98, 106803 (2007).

${ }^{9}$ J. E. Moore and L. Balents, Phys. Rev. B 75, 121306 (2007).

${ }^{10}$ J. C. Y. Teo, L. Fu, and C. L. Kane, Phys. Rev. B 78, 045426 (2008).

${ }^{11}$ C. L. Kane, Nat. Phys. 4, 348 (2008).

${ }^{12}$ A. P. Schnyder, S. Ryu, A. Furusaki, and A. W. W. Ludwig, Phys. Rev. B 78, 195125 (2008).

${ }^{13}$ X.-L. Qi, T. L. Hughes, and S.-C. Zhang, Phys. Rev. B 78, 195424 (2008).

${ }^{14}$ D. Hsieh, D. Qian, L. Wray, Y. Xia, Y. S. Hor, R. J. Cava, and M. Z. Hasan, Nature (London) 452, 970 (2008).

${ }^{15}$ L. Fu and C. L. Kane, Phys. Rev. Lett. 100, 096407 (2008).

${ }^{16}$ H. Zhang, C.-X. Liu, X.-L. Qi, X. Dai, Z. Fang, and S.-C. Zhang, Nat. Phys. 5, 438 (2009).

${ }^{17}$ M. Buttiker, Science 325, 278 (2009).

${ }^{18}$ Y. Xia, D. Qian et al., Nat. Phys. 5, 398 (2009).

${ }^{19}$ H.-J. Zhang, C.-X. Liu, X.-L. Qi, X.-Y. Deng, X. Dai, S.-C. Zhang, and Z. Fang, Phys. Rev. B 80, 085307 (2009).

${ }^{20}$ P. Roushan, J. Seo, C. V. Parker, Y. S. Hor, D. Hsieh, D. Qian, A. Richardella, M. Z. Hasan, R. J. Cava, and A. Yazdani, Nature (London) 460, 1106 (2009).

${ }^{21}$ Y. Ran, Y. Zhang, and A. Vishwanath, Nat. Phys. 5, 298 (2009).

${ }^{22}$ R. Roy, Phys. Rev. B 79, 195322 (2009).
${ }^{23}$ R. Roy, Phys. Rev. B 79, 195321 (2009).

${ }^{24}$ A. P. Schnyder, S. Ryu, and A. W. W. Ludwig, Phys. Rev. Lett. 102, 196804 (2009).

${ }^{25}$ Y. L. Chen et al., Science 325, 178 (2009).

${ }^{26}$ A. M. Essin, J. E. Moore, and D. Vanderbilt, Phys. Rev. Lett. 102, 146805 (2009).

${ }^{27}$ J. Moore, Nat. Phys. 5, 378 (2009).

${ }^{28}$ D. Hsieh et al., Science 323, 919 (2009).

${ }^{29}$ D. Hsieh, L. Wray, D. Qian, Y. Xia, Y. Hor, R. Cava, and M. Hasan, arXiv:1001.1574 (unpublished).

${ }^{30}$ A. A. Taskin and Y. Ando, Phys. Rev. B 80, 085303 (2009).

${ }^{31}$ A. Taskin, K. Segawa, and Y. Ando, Phys. Rev. B 82, 121302(R) (2010).

${ }^{32}$ Y. Hor et al., Phys. Rev. B 81, 195203 (2010).

${ }^{33}$ J. Analytis, J. Chu, Y. Chen, F. Corredor, R. McDonald, Z. Shen, and I. Fisher, Phys. Rev. B 81, 205407 (2010).

${ }^{34}$ K. Eto, Z. Ren, A. A. Taskin, K. Segawa, and Y. Ando, Phys. Rev. B 81, 195309 (2010).

${ }^{35}$ U. Fano, Phys. Rev. 124, 1866 (1961).

${ }^{36}$ For concreteness, we consider a single surface-state branch running along a domain wall in an otherwise bulk system. This is produced by a soliton mass in the appropriate continuum Dirac model for the topological insulator bulk, the surface states being the localized solutions first found in Ref. 43.

${ }^{37}$ G. D. Mahan, Many-Particle Physics (Plenum Press, New York, 1981).

${ }^{38}$ S. F. Edwards, Philos. Mag. 3, 1020 (1958).

${ }^{39}$ S. R. Park, W. S. Jung, C. Kim, D. J. Song, C. Kim, S. Kimura, K. D. Lee, and N. Hur, Phys. Rev. B 81, 041405 (2010).

${ }^{40}$ C. Xu and J. E. Moore, Phys. Rev. B 73, 045322 (2006).

${ }^{41}$ E. Abrahams, P. W. Anderson, D. C. Licciardello, and T. V. Ramakrishnan, Phys. Rev. Lett. 42, 673 (1979).

${ }^{42}$ E. Kneedler, K. E. Smith, D. Skelton, and S. D. Kevan, Phys. Rev. B 44, 8233 (1991).

${ }^{43}$ R. Jackiw and C. Rebbi, Phys. Rev. D 13, 3398 (1976). 